\title{
A qualitative secondary evaluation of statewide follow-up interviews for abnormal newborn screening results for cystic fibrosis and sickle cell hemoglobinopathy
}

\author{
Alison La Pean, MS, CGC'1, Jenelle L. Collins, BSN, RN ${ }^{1}$, Stephanie A. Christopher, MA ${ }^{1}$, \\ Kerry L. Eskra, BBA ${ }^{1}$, Sara J. Roedl, PhD ${ }^{1}$, Audrey Tluczek, PhD, RN² and Michael H. Farrell, MD ${ }^{1}$
}

\begin{abstract}
Purpose: The purpose of this qualitative analysis was to assess parental acceptability of large-scale, telephone follow-up regarding their infants' newborn screening (NBS) results, indicating carrier status for sickle cell hemoglobinopathy (SCH) and cystic fibrosis (CF).

Methods: Analysis of 195 interview transcripts focused on parents' responses to two open-ended questions: "What was your reaction to being called by me?" and "What do you think of the state NBS program having follow-up people calling parents like you?" Responses were coded using conventional content analysis procedures, and nonparametric tests were performed to analyze quantitative data.
\end{abstract}

Results: Most parents reported favorable opinions about the follow-up. Favorable opinions were associated with several emotional reactions to receiving follow-up $(P<0.001)$ and three reasons why parents found the interview beneficial $(P<0.05)$ : it provided information, clarified NBS results, and answered questions. Seventeen parents of $\mathrm{SCH}$ carriers reportedly had not been told their infant's NBS results and received them for the first time during the follow-up interview.

Conclusion: Parents of CF and SCH carrier infants had favorable opinions and identified specific benefits to receiving follow-up contact. This analysis demonstrates an information deficit among carrier parents and illustrates the importance of NBS follow-up and need for comprehensive communication and counseling.

Genet Med 2012:14(2):207-214

Key Words: cystic fibrosis carrier; follow-up counseling; newborn screening; provider-patient communication; sickle cell trait

\section{INTRODUCTION}

Nearly every infant born in the United States undergoes newborn screening (NBS) through blood samples obtained shortly after birth. This population-scale public health program screens for several conditions in order to save lives and provide early intervention and treatment to affected infants. Wisconsin's NBS program screens for 47 conditions, including two of the world's most common genetic diseases, cystic fibrosis (CF) and sickle cell hemoglobinopathy (SCH). Early identification of these conditions has proven beneficial in the prevention of infections for infants with $\mathrm{SCH}^{1}$ and nutritional deficiencies or lung problems for infants with $\mathrm{CF}^{2-4}$

The United States is at the forefront of incorporating innovation into practice as NBS has undergone a recent, rapid expansion with the advent of multiplex and DNA technologies. The high sensitivity of new screening technologies not only allows for detection of those infants affected with $\mathrm{SCH}$ and $\mathrm{CF}$ but also identifies heterozygous genetic "carriers" in the process. A carrier for a recessively inherited condition (sometimes called "trait") refers to persons with a single deleterious mutation for that genetic condition. The United States carrier frequency is estimated to be 1 in 12 for $\mathrm{SCH}$ in African Americans ${ }^{5}$ and 1 in 28 for $\mathrm{CF}^{6}$ in Caucasians.
The concept of "carrier status" can be confusing for parents to understand, and primary care providers' (PCPs') communication of these results has been criticized..$^{7-14}$ These challenges can lead to the development of parental psychosocial complications such as depression, anxiety, fear, and confusion. ${ }^{14-21}$ We contend that these consequences are potentially avoidable or alleviated by providing access to follow-up and informational resources. We therefore implemented the "Wisconsin Project on Improvement of Communication Processes and Outcomes after Newborn Screening" to serve the nearly 1000 Wisconsin families with infants found to be carriers for $\mathrm{CF}$ and $\mathrm{SCH}$ via NBS annually. ${ }^{22,23}$ This project was designed as a quality improvement effort to assess parents' experiences with their infants' NBS results disclosure for SCH and CF carriers and evaluate their opinions of NBS telephone follow-up.

Many NBS programs are interested in routine telephone follow-up for families of carrier infants, but critics charge that there is no published evidence that parents would accept such contacts from centralized public health systems. Our statewide implementation of an elaborate communication and follow-up study for families of $\mathrm{CF}$ and $\mathrm{SCH}$ carrier infants provided an excellent opportunity to evaluate parental acceptability of routine, telephone contact for a centralized program that used 
public health follow-up techniques. In addition to using traditional evaluation surveys, ${ }^{23}$ we decided to incorporate two open-ended acceptability questions into our contacts with parents because of the limitations of fixed-choice and close-ended questions. This alleviated potential bias effects often seen in traditional evaluation surveys and allowed parents to provide feedback on any aspect of the follow-up, rather than confining their opinions to specific topics. The purpose of this qualitative, secondary analysis of open-ended interview questions was to assess parental acceptability of large-scale, statewide telephone follow-up regarding their infants' NBS results.

\section{MATERIALS AND METHODS}

\section{Study design}

This article describes findings of a qualitative, secondary analysis from statewide follow-up telephone interviews with parents after NBS. Analysis focused on parents' responses to two openended acceptability questions:

Question 1: What was your reaction to being called by me? Question 2: What do you think of the state NBS program having follow-up people like me calling parents like you?

The goal of this analysis was to examine parents' reactions to NBS follow-up in a richer and more descriptive fashion than is possible with more traditional, Likert-scaled evaluation surveys.

\section{Data source: the Project}

This analysis used parent interview data from the "Wisconsin Project on Improvement of Communication Processes and Outcomes after Newborn Screening" (henceforth called "the Project"), which represents a collaboration of the Wisconsin State Laboratory of Hygiene (WSLH) and the Department of Health Services with the Medical College of Wisconsin as contracted Project agent. This Project was designed as both a research study and a statewide quality improvement initiative to serve families whose infants were found to be heterozygous genetic carriers for $\mathrm{SCH}$ or $\mathrm{CF}^{23}$ The Project aimed to assess parents' psychosocial outcomes after NBS results disclosure and provide brief counseling to parents if needed; however, this report describes only parents' responses to the two acceptability questions evaluating the follow-up interview itself. Forthcoming manuscripts will discuss results of the larger Project, including parents' experiences and psychosocial outcomes related to NBS results disclosure. ${ }^{23}$ The Project is consistent with the National Institutes of Health criteria for waiver of consent for initial contact procedure. ${ }^{24}$ The Project was authorized by Department of Health Services and approved by Institutional Review Boards at the Medical College of Wisconsin and University of Wisconsin-Madison.

\section{Participants and recruitment}

Nearly every infant born in Wisconsin undergoes NBS and specimens are sent to WSLH for analysis. Project participants included parents and PCPs of infants found via NBS to be carriers for SCH or CF. SCH carrier status for the Project was defined as the presence of fetal, adult, and sickle hemoglobin ("F-A-S") on NBS. Other heterozygous results for hemoglobinopathy (e.g., "F-S-A") were excluded to minimize confounding effects for the Project's primary analyses. CF carrier status was defined as a screening result that showed elevated immunoreactive trypsinogen and a single CFTR mutation, followed by a normal sweat chloride test. The CF Foundation recommends all infants with abnormal CF screening results undergo sweat chloride testing because $2-5 \%$ may have a second unidentified mutation, not included in the Wisconsin NBS panel, ${ }^{25,26}$ resulting in CF the disease. Only confirmed CF carriers, with normal sweat chloride test results, were considered eligible for the Project.

Parents of infants were enrolled in the Project as follows. NBS results were faxed from WSLH to the Project's study team for infants with results indicating F-A-S or elevated immunoreactive trypsinogen with a single CFTR mutation. Infants were immediately excluded based on the following additional criteria: (1) infant had more than one abnormal NBS result, indicative of a coexisting condition; (2) infant's parent had another child previously enrolled and declined to participate; and (3) infant considered "medically fragile" defined as $<35$ weeks gestation, spending $>5$ days in nursery or neonatal intensive care, or being rehospitalized. Infants were also excluded if their PCP identified any contraindication to the Project's telephone follow-up interview, such as an English-language barrier or psychosocial issues deemed problematic for additional contact and follow-up. Finally, infants were excluded based on sweat chloride testing that had ambiguous results, was performed on infants $>5$ months old, or resulted in a CF diagnosis.

Recruitment procedures involved two steps. First, when infants were 3-5 months old, parents were sent recruitment mailings containing an explanation of the Project and a "decline of contact" card. This procedure allowed parents to decline the Project contact without becoming fully informed about the purpose of the research. To avoid parental confusion or distress if, perhaps, parents had not been informed or were confused about their infants' NBS results, recruitment mailings did not mention infants' NBS results. The second step in the recruitment process occurred approximately 10 days after recruitment materials had been mailed to parents. If we had not received "decline of contact" cards, research team members telephoned parents inviting them to participate in a one-time telephone interview about the disclosure of their infant's NBS results and psychosocial outcomes. Parents who participated in the follow-up interview received a $\$ 20$ gift certificate in gratitude for their time.

\section{Description of the interview}

The interview questions and script were developed by a team with methodological and content expertise in pediatric medicine, NBS, genetic counseling, nursing, communication, and bioethics. Parents participated in a one-time, follow-up telephone interview which lasted approximately 20-30 minutes, 
depending on parent response. Interviews began with informed consent, followed by a carefully scripted, directive, semistructured discussion balancing fixed-choice and open-ended research questions. The directive nature of the interview script ensured consistent data collection on a set of predetermined questions, particularly useful with such a large study population; however, the semistructured approach also allowed for additional unscripted probing questions to clarify parent responses and prompt further elaboration when necessary. The semistructured approach also allowed interviewers to provide counseling in the form of parent education and emotional support, if needed. At the end of the interview, after all other research questions were asked and all counseling was provided, if necessary, the two open-ended acceptability questions were asked about parents' opinions of the Project's follow-up.

Interviews were conducted by one member of a multidisciplinary team comprised of nurses, a physician, and a genetic counselor. Before the Project's start date, it was anticipated that the parent interviewers would potentially be contacting parents who had not heard their infant's NBS results. Because the follow-up interview with parents of CF carriers happened after the infant's sweat test, where the infant was confirmed a carrier, the study team could be certain that these parents had been informed of their infant's results, likely by more than one provider. Alternatively, we anticipated that any follow-up contact requiring results disclosure would likely happen with parents of SCH carriers, as their PCP would be the primary source for disclosing their infant's NBS results. Because of the definitive nature of the $\mathrm{SCH}$ screening, these results are not ambiguous and do not require additional testing for diagnostic purposes (unlike CF carrier infants). Given the potential that some parents may not have been informed of their infant's NBS results, all interviewers had a clinical background and experience delivering clinical test results or diagnoses to parents in a pediatric setting. All interviewers were trained to assess signs of emotional distress or confusion. If either occurred, the interviewee or the interviewer had the option of forgoing the research questions, making the interview a purely clinical intervention. Both research and clinical calls ended with debriefing to ensure misconceptions identified during the interview were clarified and parents knew who to contact with additional questions. Interviews were audio-recorded, transcribed, proofread, and deidentified as they occurred.

\section{Data management and sample}

Between December 2007 and August 2010, NBS results for 1,781 infants were faxed to the Project's study team by WSLH. Of these, 617 infants met immediate exclusion criteria (as described earlier). Recruitment letters were mailed to the remaining 1,164 parents, 11 parents returned a "decline of contact" card, and so 1,153 parents were contacted by telephone to participate. Of these, 144 parents verbally declined to participate, 548 had not responded to the recruitment telephone calls by the start of this analysis, and 461 chose to participate in the follow-up interview. When this analysis began, 175 interviews were unavailable for use and therefore excluded because of transcription delays, audio recording failures, or because they became clinical interventions. Of the remaining 286 transcripts, 195 included parents' responses to at least one of the acceptability questions.

The final set of 195 transcripts consisted of interviews with 190 mothers and 5 fathers. Two thirds were parents of SCH carriers while one third were parents of CF carriers (Table 1), an identical proportion to that found in the total interviewed sample $(n=461)$ of the Project. Although most parents of sickle cell trait infants reported their race/ethnicity as African American $(n=90)$, some identified themselves as Latino $(n=7)$, Caucasian $(n=26)$, or another race $(n=7)$. Similarly, most parents of CF carriers identified themselves as Caucasian $(n=53)$, although some reported their race/ethnicity as African American $(n=7)$ or another race $(n=5)$.

\section{Analysis}

Parents' responses to the two open-ended acceptability questions were extracted from each transcript to form the dataset for this analysis. Extracted responses were coded following an adapted version of conventional content analysis procedures. ${ }^{27}$ The team conducted line-by-line, open coding on a subset of data to identify categories and develop a coding schema. ${ }^{27-30}$ Coders compared their impressions of key concepts found in the text until no new themes emerged from the dataset. Data describing similar ideas were clustered into themes and organized into subthemes, labeled with descriptive codes, and arranged into a codebook. ${ }^{28,31,32}$ Disagreements about coding

\section{Table 1 Participant demographics}

\begin{tabular}{lc} 
Variable & $\boldsymbol{n}(\mathbf{\%})$ \\
\hline Parent gender & $190(97.4)$ \\
\hline Female & $5(2.6)$ \\
\hline Male & \\
\hline Parent race/ethnicity & $92(47.2)$ \\
\hline Black & $79(40.5)$ \\
\hline White & $6(3.1)$ \\
\hline Latino & $12(6.2)$ \\
\hline Other & $6(3.1)$ \\
\hline Not asked & \\
\hline Parent age (years) & $22(11.3)$ \\
\hline$<20$ & $114(58.5)$ \\
\hline 20-29 & $51(26.2)$ \\
\hline $30-39$ & $3(1.5)$ \\
\hline $40-49$ & $5(2.6)$ \\
\hline Not asked & $130(66.7)$ \\
\hline Infant's carrier status & $65(33.3)$ \\
\hline Carrier for sickle cell disease (trait) & \\
\hline Carrier for cystic fibrosis & \\
\hline Cystic fibrosis carier infants & \\
\hline
\end{tabular}

${ }^{a}$ Cystic fibrosis carrier infants in this analysis had an elevated immunoreactive trypsinogen, a single CFTR mutation, and a normal sweat chloride test. 
were resolved through discussion, consensus ratings, and revisions of the codebook. A second subset of data were coded to establish the utility of the coding system, and the codebook was revised and refined as needed. The final version of the codebook contained 3 major themes, 4 subthemes, and 62 codes describing parents' responses to the acceptability questions.

Coding of the entire data set using NVivo 8 software was performed in rounds. In each round, the 4 members of the coding team individually coded 10 transcripts apiece, followed by consensus coding of an additional 10 transcripts. Consensus coding began with two team members coding a single transcript, and then group consensus meetings were held to compare codes with the entire team to promote discussion, avoid coding drift, and intermittently evaluate intercoder reliability. As a result of these rounds, a randomly selected $80 \%$ of transcripts were individually coded, while the remaining $20 \%$ were coded by group consensus. Each individual sentence was considered a "unit" for coding and analysis, as subjectively identified by the transcriptionist based on grammar and breaths. Codes represented specific words verbalized by parents during their answer to the interview questions and were incorporated into the development of the coding schema. Sentences were coded as neutral when parents' reactions or opinions did not align with any of the positive/favorable, negative/unfavorable, surprised, or confused codes and could not be classified objectively. Quantitative analysis was conducted to better understand the associations between the emergent themes identified in the dataset. NVivo output in the form of a "coding summary report" was transformed into a spreadsheet indicating the presence or absence of codes within transcripts. Nonparametric tests were performed using SPSS.

\section{RESULTS}

This section describes parents' responses to two open-ended acceptability questions, with descriptive quotations. Results revealed three main themes: parents' opinions about the Project's follow-up interview, reasons why parents found the Project's follow-up interview beneficial, and parents' emotional reactions to the Project's follow-up interview. Because of the qualitative nature of this analysis, not all parents commented on all themes.

\section{Theme 1: parents' opinions about the Project's follow-up interview}

Of the 195 parents' responses included in this analysis, most contained at least one comment about their general opinions about the Project's follow-up interview $(n=172 / 195,88 \%$; parents of CF carriers 59/65, 91\% and SCH carriers $113 / 130$, $87 \%)$. Further details about the reasons for these opinions are presented under Theme 2 later.

Most parents reported only favorable opinions of the Project's follow-up interview ( $n=141 / 195,72 \%$; parents of CF carriers $48 / 65,74 \%$ and SCH carriers 93/130, 72\%). Sentences were coded as "favorable" when parents' responses were considered encouraging, complimentary, or implied overall merit, support, or approval of receiving follow-up from the Project.
"Um, I actually was glad that I got the letter because I do think there is a lot more education that needs to be done about newborn screening so I was glad that, you know, that there's a whole project looking into, you know, just bettering the program...I think that's a good idea because I think there are probably a lot of people who don't fully understand it ..." (mother, CF carrier infant)

A code for unfavorable opinion of the follow-up interview was developed but remained unused. One parent of a CF carrier reported general confusion or uncertainty as their only opinion.

Some parents expressed only neutral opinions about the Project's follow-up interview ( $n=11 / 195,6 \%$; parents of CF carriers 5/65, 8\% and SCH carriers 6/130, 5\%). Sentences such as "I think it's okay" or "I don't see nothing bad about it" were coded as neutral. A minority of parents reported combinations of favorable, neutral, or confused opinions about the Project $(n=19 / 195,10 \%$; parents of CF carriers $5 / 65,8 \%$ and SCH carriers $14 / 130,11 \%)$.

Theme 2: reasons why parents found the Project's followup interview beneficial

Many parents ( $n=120 / 195,62 \%$; parents of CF carriers $33 / 65$, $51 \%$ and SCH carriers $87 / 130,67 \%$ ) reported at least one reason they found the follow-up interview beneficial. More than half of these 120 parents reported two or more reasons $(n=$ 64/195, 34\%; parents of CF carriers $21 / 65,32 \%$ and SCH carriers $43 / 130,33 \%)$. The five most commonly reported reasons are listed in Table 2. The most frequent reason reported was that the follow-up was an opportunity to obtain additional information about such topics as genetics or general NBS information. Parents also reported benefit to receiving an explanation or further clarification about their infants' NBS results, especially when they were confused about the meaning of the results. Consequently, several parents reported an increased understanding about the NBS results. Parents considered the interview an opportunity to obtain answers to their questions not addressed at the time of NBS results disclosure or that arose later. For many parents, the interview reinforced NBS information they had already heard from their infant's provider or reassured them that their infant was healthy. Finally, parents reasoned that the interview was beneficial because it offered additional resources and emotional support.

Some parents reportedly had not been informed about their infant's NBS results by the PCP and received them for the first time during the follow-up interview ( $n=17 / 130,13 \%$ parents of SCH carriers only). For them, the interview represented their only source of information about the NBS results. Most of these parents $(n=15)$ reported this as a beneficial reason for the follow-up interview. Some parents chose not to answer any of our research questions upon hearing their infant's results and requested that the call become a clinical intervention, where the interviewer provided counseling to parents. These interviews were not recorded and therefore do not have transcripts available for analysis. Because no transcripts were available for 
Table 2 Reasons why parents found the Project's follow-up interview beneficial

\begin{tabular}{|c|c|c|c|c|}
\hline Reason & Total \% (n) & $\mathrm{SCH} \%(n)$ & CF \% (n) & Quote \\
\hline $\begin{array}{l}\text { Provided } \\
\text { information }^{\text {a }}\end{array}$ & $26 \%(51 / 195)$ & $31 \%(40 / 130)$ & $17 \%(11 / 65)$ & $\begin{array}{l}\text { "... I'm happy because that was something that I actually wish that I could've } \\
\text { talked to somebody about because I felt like, you know, I know that there's } \\
\text { other parents going through this, too, and it would've been really nice to } \\
\text { have more information especially right up front." (mother, CF carrier infant) }\end{array}$ \\
\hline $\begin{array}{l}\text { Clarified results } \\
\text { or increased } \\
\text { understanding }\end{array}$ & $23 \%(44 / 195)$ & $25 \%(33 / 130)$ & $17 \%(11 / 65)$ & $\begin{array}{l}\text { "Yes, 'cause as you said its probably a lot of people that don't understand } \\
\text { the difference in the trait and the disease and I don't even really understand } \\
\text { the ... the ... how they [PCP] were breaking down the likelihood of if we } \\
\text { had more kids you know the chances of that kid getting the disease and not } \\
\text { the trait so it gets even more confusing for me so ... um and a lot of people } \\
\text { probably won't reach out and ... and ask so it's I think is good that you're } \\
\text { seeking out the parents to make sure that they do understand." (mother, } \\
\text { SCH carrier infant) }\end{array}$ \\
\hline $\begin{array}{l}\text { Answered } \\
\text { questions }^{\text {a }}\end{array}$ & $18 \%(35 / 195)$ & $16 \%(21 / 130)$ & $22 \%(14 / 65)$ & $\begin{array}{l}\text { "I think that there's a lot of us out here who have questions and sometimes } \\
\text { we don't get the answers that we need, or we don't have the time to get } \\
\text { the answers to the questions that we have, so if we can just sit down with } \\
\text { people and, you know, ask questions and have them answer them for us } \\
\text { and understand and, you know, see where we're coming from, that's a great } \\
\text { thing." (mother, CF carrier infant) }\end{array}$ \\
\hline $\begin{array}{l}\text { Received results } \\
\text { for first time }\end{array}$ & $8 \%(15 / 195)$ & $12 \%(15 / 130)$ & $\mathrm{n} / \mathrm{a}$ & $\begin{array}{l}\text { "I like [the follow-up call] very much because before you called me I didn't } \\
\text { even know he had the trait ... So ... I ... I do appreciate [the study team] } \\
\text { taking the time out to call new ... new mothers like myself 'cause I didn't } \\
\text { know anything about this at all or the results." (mother, SCH carrier infant) }\end{array}$ \\
\hline
\end{tabular}

CF, cystic fibrosis; n/a, not applicable; $\mathrm{SCH}$, sickle cell hemoglobinopathy.

"SCH" refers to parents of SCH carrier infants; " $\mathrm{CF}$ " refers to parents of CF carrier infants; percents are rounded to the nearest whole percent.

${ }^{a}$ Associated with (Theme 1) favorable opinion of the Project $(P<0.05)$. ${ }^{b}$ Associated with (Theme 1) favorable opinion of the Project $(P<0.001)$.

this set of parents, interview notes were reviewed to find all instances of results disclosure. By the start of our analysis in August 2010, the study team had completed interviews for 461 parents, to include 306 parents of SCH carriers, and informed an additional 30 parents of their infant's SCH carrier results (47 parents in total). This totals 15\% (47 of the 306 interviews) of parents participating as part of this quality improvement Project.

\section{Theme 3: parents' emotional reactions to the Project's follow-up interview}

Most parents ( $n=174 / 195$, 89\%; parents of CF carriers 60/65, 92\% and SCH carriers 114/130, 88\%) expressed an initial emotional reaction to receiving follow-up from the Project's team. Emotional reactions were organized into four subthemes: positive, negative, surprise, and neutral or no reaction (Table 3). Some parents described more than one emotional reaction $(n=29 / 195,15 \%$; parents of CF carriers $11 / 65,17 \%$ and SCH carriers $18 / 130,14 \%$ ), in which case each reaction was coded separately.

Parents mentioned positive emotional reactions to receiving the follow-up that included feeling happy, reassured, comforted, relieved, excited, and appreciative $(n=27 / 195,14 \%$; parents of CF carriers 8/65, 12\% and SCH carriers 19/130, 14\%). Although most parents described their positive reactions to our interview with a specific emotion, several parents simply stated they had a "good" or "nice" reaction generally.
Negative emotional reactions were occasionally mentioned $(n=2 / 195,1 \%$; parents of CF carriers $1 / 65,2 \%$ and SCH carriers $1 / 130,<1 \%)$. The most common negative emotions were feeling anxious, scared, or worried. Two parents attributed these emotions to not expecting our telephone contact. Some parents initially assumed that we were calling to discuss a different, previously undisclosed NBS result. This resulted in initial anxiety or fear, which was alleviated once the Project's study team clarified the reason for the call at the beginning of the interview. In two instances, parents expressed initial annoyance with our follow-up calls because they assumed we were telemarketers.

"Well, at first I thought you were a telemarketer so I was going to read you off. But then you said, you know, who you were, you know, what you were calling about and then I was like, Okay, all right, that's fine, didn't have to tell you off?" (mother, CF carrier infant)

Some parents reported feeling surprised by our follow-up interview $(n=12 / 195,6 \%$; parents of CF carriers $4 / 65,6 \%$ and SCH carriers $8 / 130,6 \%$ ). Of these, several commented that they had not been expecting our telephone call and they did not remember receiving our recruitment letter. Other parents reported surprise because of the time lapse (approximately 3-5 months) between the NBS process and the follow-up interview, or they were unaware that research is being done to follow-up with parents after NBS.

Many parents expressed a neutral reaction or stated they did not have a reaction to participating in the Project's follow-up 


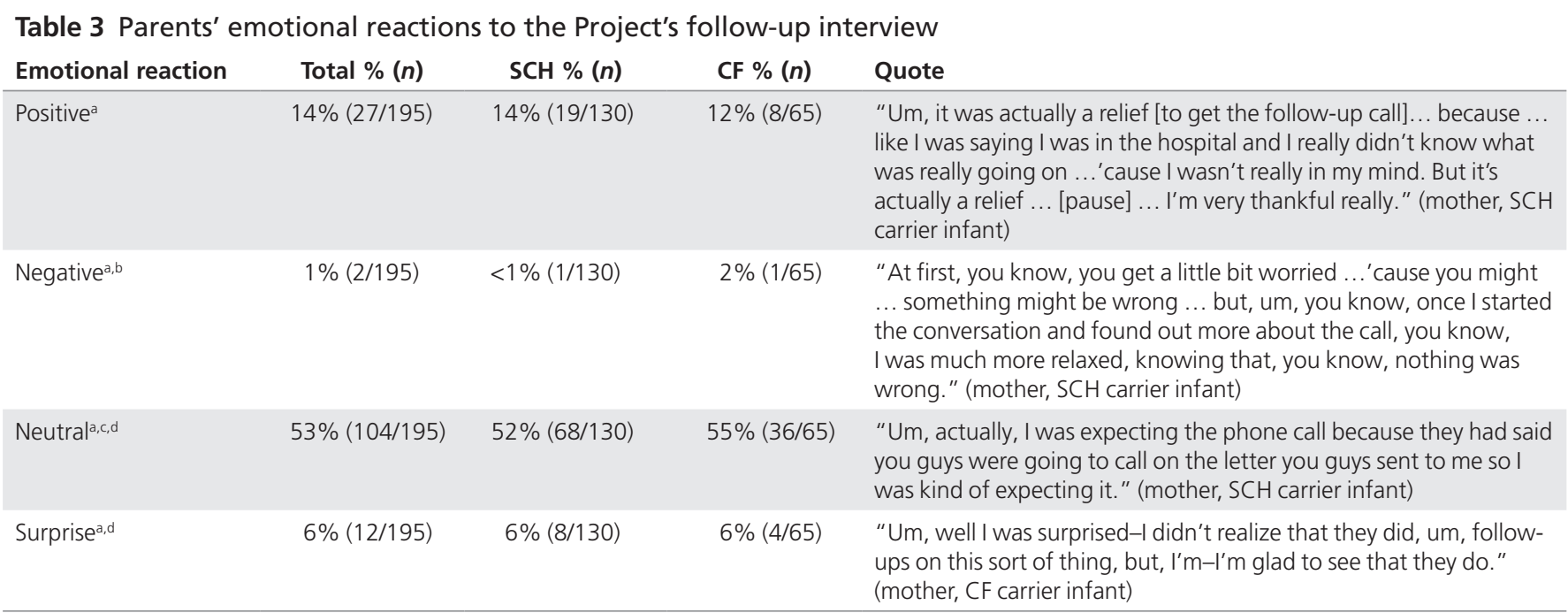

"SCH" refers to parents of SCH carrier infants; "CF" refers to parents of CF carrier infants; percents are rounded to the nearest whole percent.

associated with (Theme 1) favorable opinion about the follow-up interview $(P<0.001)$. ${ }^{b}$ Associated with (Theme 2 ) reporting a reason for finding the interview beneficial $(P<0.05)$. 'Associated with (Theme 1$)$ neutral opinion about the follow-up interview $(P<0.05)$. ${ }^{d}$ Associated with (Theme 2$)$ reporting a reason for finding the interview beneficial $(P<0.001)$.

interview such as "I felt fine," "it didn't bother me," or "I didn't have a reaction" ( $n=104 / 195,53 \%$; parents of CF carriers $36 / 65,55 \%$ and SCH carriers 68/130,52\%). Many of these parents commented that they were expecting our call because they remembered the recruitment letter or voicemails from the Project's study team.

\section{Quantitative analysis}

Associations between the three themes are marked in Tables 2 and 3 with distinct symbols. We tested for associations between parents' emotional reactions (Theme 3) and their opinions about the Project's follow-up interview (Theme 1). All categories of emotional reactions (positive, negative, neutral, and surprised) were associated with favorable opinions of the followup interview $(P \leq 0.001)$. Only parents with neutral emotional reactions were likely to have neutral opinions about the followup interview $(P<0.05)$.

We also found associations between overall opinions about the follow-up interview (Theme 1) and parents' reasons for finding the follow-up interview beneficial (Theme 2). The following three reasons were significantly associated with favorable opinions about the follow-up interview $(P<0.05)$ : providing information, clarifying NBS results, and answering questions (Table 2). No association was found between neutral opinions about the follow-up interview and reporting a reason for finding the interview beneficial.

Parents' emotional reaction to the follow-up interview (Theme 3 ) was associated with (Theme 2) reporting a reason for finding the interview beneficial (Table 3). Parents with neutral, negative, and surprised reactions were more likely to report a reason for finding the follow-up interview beneficial $(P<0.05)$. Parents with positive reactions were not significantly associated with finding the follow-up interview beneficial in this secondary analysis $(P=$ 0.077); however, the data show a trend toward significance.

\section{DISCUSSION}

This qualitative, secondary analysis of interview acceptability questions is the first report of its kind that addresses acceptability of telephone follow-up regarding NBS carrier results. Our data from parent responses to open-ended interview questions show that routine telephone follow-up after NBS is equally acceptable to parents of both $\mathrm{SCH}$ and $\mathrm{CF}$ carriers. Indeed, the majority of parents interviewed (72\%) had favorable opinions of the follow-up interview and many parents (62\%) reported reasons why they found the follow-up beneficial.

Parents' emotional reactions to receiving follow-up contact were reported among both groups of parents proportionally, and all reactions were significantly associated with an overall favorable opinion of the follow-up contact. It should be noted that even parents with initial neutral, negative, or surprised reactions were significantly likely to identify reasons they found the interview beneficial, while a nonsignificant trend for finding the follow-up interview beneficial was found among parents who reported an initial positive reaction. We hypothesize that these parents with positive reactions already had sufficient information and understanding about their infant's NBS results and therefore did not need further counseling or clarification from the interviewer. This potentially minimized their perceived benefit from the follow-up contact.

The data suggest that parents of carriers for $\mathrm{CF}$ and $\mathrm{SCH}$ found the follow-up calls beneficial but perhaps for different reasons. For CF carrier parents, a larger proportion reported that the interview provided them with another resource and an opportunity to have questions answered that either were not addressed by PCPs at initial results disclosure or specialists upon sweat testing. Perhaps, new questions arose in the interim between results disclosure and the follow-up interview.

Alternatively, a larger proportion of SCH carrier parents reported that the interview was beneficial because it provided 
them with additional information and increased their understanding by clarifying the NBS results. Often, parents wanted more information about inheritance, especially regarding the possibility of carrier status in themselves and family members. Other parents were confused about the differences between $\mathrm{SCH}$ trait and SCH disease and the implications of these differences such as questioning whether trait could become disease in the future.

Perhaps the most noteworthy finding in this analysis was among parents of SCH carriers. The follow-up interview served as the primary source of information for $12-15 \%$ of parents regarding their infants' status as SCH carriers. There are several reasons parents may not have heard results. Parents may have changed PCPs or clinics, and so the result would have been sent to the incorrect location, or they may have missed appointments when the results would have been discussed. On several occasions throughout this study, our team found that a simple NBS card omission such as the infant's first name could lead to confusion, or if the infant's name had changed from the time the baby was screened in the hospital (i.e., NBS card was submitted with mom's last name "baby boy Smith," but infant's full name was actually "John Jones"). It is possible that another family member knew the infant's NBS results other than the parent interviewed (i.e., mom took infant to results disclosure appointment, but our study team interviewed dad). Some parents reported thinking that their infant's NBS results were mailed to them in a formal letter, but they never read the letter. Finally, it may be possible that the infant's parent was actually told the NBS result but did not remember hearing it.

The healthcare expectation in pediatric and family medicine is that all parents are told their infant's NBS results, provided adequate information, and offered necessary counseling by PCPs (and later by other health professionals, in the case of CF). We were surprised to find that such a large portion of parents interviewed had either not been told their infant's NBS results or had lingering concerns, questions, or knowledge gap. Even with PCPs doing their best to inform parents of their infant's NBS results, these findings suggest that our healthcare system may not adequately meet the informational needs of this population. Furthermore, this information deficit illustrates the importance of NBS follow-up and the need for comprehensive NBS communication and counseling.

There are several limitations to our analysis. First, interview participation was potentially subject to self-selection bias. Only parents wanting to participate in the Project's follow-up interview completed the interview, possibly skewing data toward favorable opinions and feedback. Second, social desirability may have influenced parents' responses to our interview questions. It is possible that participants did not want to report negative opinions to the interviewer, skewing data toward favorable responses. Finally, PCPs may have introduced bias by advising against inclusion of families meeting the Project's contraindications. Most contraindications identified were language barriers (non-English speaking), and only a few families were identified as having "psychosocial" issues. In fact, PCPs were mostly encouraging of the Project's follow-up interview for families with possible "psychosocial" issues, noting the benefits of additional attention and follow-up.

Despite these limitations, the clinical implications of these data suggest the importance for PCPs to fully disclose NBS carrier results to all parents. In addition, access to clinical follow-up after initial results disclosure is valuable and relevant to parents regarding their infant's $\mathrm{CF}$ or $\mathrm{SCH}$ carrier status, perhaps via telephone or at subsequent PCP appointments. Although telephone follow-up from the statewide NBS program may be impractical, clinical follow-up by PCPs or other clinical professionals may be more feasible and can provide parents an opportunity to ask additional questions and access further information.

\section{CONCLUSION}

Routine telephone follow-up for parents of CF and SCH carriers identified via NBS is both feasible and acceptable to parents. Most parents interviewed had favorable opinions and identified specific benefits to receiving the follow-up contact. Furthermore, this analysis demonstrates a potential information deficit among carrier parents and illustrates the importance of NBS follow-up and the need for comprehensive NBS communication and counseling. Future studies of the larger Project will examine these informational deficits, the need for clinical follow-up, and the gaps in reporting NBS results.

\section{ACKNOWLEDGMENTS}

The Project is funded by NIH grants K01-HL072530 and R01HL086691. The authors acknowledge Hollie Beaudry, RN, and Jill Paradowski, RN, MS, for their contributions to data collection. The authors also acknowledge the families of carriers for sickle cell hemoglobinopathy and cystic fibrosis whose participation made this manuscript possible.

\section{DISCLOSURE}

The authors declare no conflict of interest.

\section{REFERENCES}

1. Pass K, Harris K, Lorey F, Choi R, Kling S. Update: newborn screening for sickle cell disease-California, Illinois, and New York, 1998. MMWR 2000;49:729-731.

2. Salvatore $D$, Buzzetti $R$, Baldo $E$, et al. An overview of international literature from cystic fibrosis registries 2. Neonatal screening and nutrition/growth. J Cyst Fibros 2010;9:75-83.

3. Farrell PM, Kosorok MR, Rock MJ, et al. Early diagnosis of cystic fibrosis through neonatal screening prevents severe malnutrition and improves long-term growth. Wisconsin Cystic Fibrosis Neonatal Screening Study Group. Pediatrics 2001;107:1-13.

4. Farrell PM, Lai HJ, Li Z, et al. Evidence on improved outcomes with early diagnosis of cystic fibrosis through neonatal screening: enough is enough! J Pediatr 2005; 147(suppl 3):S30-\$36.

5. Tsaras G, Owusu-Ansah A, Boateng FO, Amoateng-Adjepong Y. Complications associated with sickle cell trait: a brief narrative review. Am J Med 2009;122:507-512.

6. Hamosh A, FitzSimmons SC, Macek M Jr, Knowles MR, Rosenstein BJ, Cutting GR. Comparison of the clinical manifestations of cystic fibrosis in black and white patients. J Pediatr 1998;132:255-259. 
7. Hadlow J, Pitts M. The understanding of common health terms by doctors, nurses and patients. Soc Sci Med 1991;32:193-196.

8. Beckman HB, Frankel RM. The effect of physician behavior on the collection of data. Ann Intern Med 1984;101:692-696.

9. Marvel MK, Epstein RM, Flowers K, Beckman HB. Soliciting the patient's agenda: have we improved? JAMA 1999;281:283-287.

10. Ley P. Communicating With Patients: Improving Communication, Satisfaction and Compliance. Croom Helm: London, 1988.

11. Miller FA, Paynter M, Hayeems RZ, et al. Understanding sickle cell carrier status identified through newborn screening: a qualitative study. Eur J Hum Genet 2010;18:303-308.

12. Cavanagh L, Compton CJ, Tluczek A, Brown RL, Farrell PM. Long-term evaluation of genetic counseling following false-positive newborn screen for cystic fibrosis. J Genet Couns 2010;19:199-210.

13. Tluczek A, Koscik RL, Modaff P, et al. Newborn screening for cystic fibrosis: parents' preferences regarding counseling at the time of infants' sweat test. J Genet Couns 2006;15:277-291.

14. Lang CW, McColley SA, Lester LA, Ross LF. Parental understanding of newborn screening for cystic fibrosis after a negative sweat-test. Pediatrics 2011;127:276-283.

15. Robertson EF, Hill GN, Pollard AC. Evaluation of a state-wide neonatal screening programme. Med J Aust 1979;1:365-367.

16. Parsons EP, Clarke AJ, Hood K, Lycett E, Bradley DM. Newborn screening for Duchenne muscular dystrophy: a psychosocial study. Arch Dis Child Fetal Neonatal Ed 2002;86:F91-F95.

17. Rothenberg MB, Sills EM. latrogenesis: the PKU anxiety syndrome. J Am Acad Child Psychiatry 1968;7:689-692.

18. Gurian EA, Kinnamon DD, Henry JJ, Waisbren SE. Expanded newborn screening for biochemical disorders: the effect of a false-positive result. Pediatrics 2006;117:1915-1921.

19. Sorenson JR, Levy HL, Mangione TW, Sepe SJ. Parental response to repeat testing of infants with 'false-positive' results in a newborn screening program. Pediatrics 1984;73:183-187.
20. Tluczek A, Koscik RL, Farrell PM, Rock MJ. Psychosocial risk associated with newborn screening for cystic fibrosis: parents' experience while awaiting the sweat-test appointment. Pediatrics 2005;115:1692-1703.

21. Beucher J, Leray E, Deneuville E, et al. Psychological effects of false-positive results in cystic fibrosis newborn screening: a two-year follow-up. J Pediatr 2010;156:771-6, 776.e1.

22. National Newborn Screening and Genetics Resource Center. National Newborn Screening Information System (NNSIS) 2008 report and ongoing reports from 2010. http://nnsis.uthscsa.edu. Accessed 1 February 2011.

23. Farrell $\mathrm{MH}$, Christopher SA, Tluczek A, et al. Improving communication between doctors and patients after newborn screening. Wisconsin Med J 2011;110:221-227.

24. Department of Health and Human Services, Code of Federal Regulations, Title 45 Public Welfare, Part 46 Protection of Human Subjects. http:// www.hhs.gov/ohrp/policy/ohrpregulations.pdf. Accessed 11 June 2011.

25. Comeau AM, Accurso FJ, White TB, et al. Guidelines for implementation of cystic fibrosis newborn screening programs: Cystic Fibrosis Foundation workshop report. Pediatrics 2007;119:e495-e518.

26. Wisconsin State Laboratory of Hygiene, Health Professionals Guide to Newborn Screening, Cystic Fibrosis. http://www.slh.wisc.edu/newborn/ guide/cystic fibrosis.dot. Accessed 14 March 2011.

27. Hsieh HF, Shannon SE. Three approaches to qualitative content analysis. Qual Health Res 2005;15:1277-1288.

28. Morgan DL. Qualitative content analysis: a guide to paths not taken. Qual Health Res 1993;3:112-121.

29. Miles MB, Huberman AM. Qualitative Data Analysis: An Expanded Sourcebook. Sage: Thousand Oaks, CA, 1994.

30. Morse JM, Field PA. Qualitative Research Methods for Health Professionals, 2nd edn. Sage: Thousand Oaks, CA, 1995.

31. Coffey A, Atkinson P. Making Sense of Qualitative Data: Complimentary Research Strategies. Sage: Thousand Oaks, CA, 1996.

32. Patton MQ. Qualitative Research and Evaluation Methods. Sage: Thousand Oaks, CA, 2002. 